\title{
Abnormal Tactile Experience Early in Life Disrupts Active Touch
}

\author{
George E. Carvell1 and Daniel J. Simons² \\ ${ }^{1}$ Department of Physical Therapy, School of Health and Rehabilitation Sciences, and 2Department of Neurobiology, \\ School of Medicine, University of Pittsburgh, Pittsburgh, Pennsylvania 15261
}

The importance of early tactile experience in the development of discriminative somatomotor function was assessed by examining the proficiency and movement strategies of rats raised without normal sensory inputs provided by their mystacial vibrissae. Infant-trimmed animals had their whiskers clipped daily from birth to $45 \mathrm{~d}$ of age, after which they were allowed to regrow for 60-70 d before initiation of behavioral training, which lasted as long as several months. Adult-trimmed animals had their whiskers trimmed for comparable periods during adulthood. Rats were tested on one of two tactile discriminations, rough versus smooth or rough versus rough, that differed with respect to the overall size of their surface features. Whisker movements during task performance were examined in detail using video-based motion analysis software. Infant-trimmed animals performed rough versus smooth discriminations as well as adult-trimmed rats or normally reared animals. Except for one subject, infant-trimmed rats were severely impaired in their ability to distinguish rough versus rough surfaces. Deficits persisted in spite of months of training with the regrown vibrissae. The animals that failed to master this task displayed whisking patterns that notably lacked frequencies in the normal range of $6-12 \mathrm{~Hz}$. Thus, abnormal tactile experience early in life substantially, and perhaps permanently, impairs sensorimotor integration underlying active touch.

Key words: active touch; tactile discrimination; vibrissa; critical period; sensory deprivation; somatosensory; sensory motor integration
The organization of cerebral cortex is influenced by sensory experience early in life. For example, a brief period of eyelid closure in young cats and primates reduces the number of visual cortical neurons having binocular properties (Hubel and Wiesel, 1970; Hubel et al., 1977), and the resulting alterations in visual perception are known to be permanent (Mitchell and Timney, 1984). Long-lasting changes in the receptive field properties of somatosensory cortical neurons have also been reported after early tactile deprivation. Whisker trimming in young rats leads to enlarged receptive fields and weaker inhibitory interactions among neighboring vibrissae (Simons and Land, 1987; Simons et al., 1993). Receptive field abnormalities persist despite months of whisker regrowth during adulthood. Experience-dependent regulation of glutamic acid decarboxylase (GAD) in whisker-related cortical barrels is also abnormal in neonatally deprived rats (Akhtar and Land, 1991). GAD levels are reduced after whisker removal in adult animals raised with a normal whisker field but not in adults whose whiskers were trimmed during the first 2 months of life. This abnormality in GAD regulation is observed even if, before whisker removal, the neonatally clipped whiskers are allowed to regrow for 2 months during adulthood (Akhtar and Land, 1991). Continued whisker plucking without complete regrowth also produces cortical receptive field abnormalities that are more pronounced in animals having their whiskers removed at earlier postnatal ages (Fox, 1992).

\footnotetext{
Received Aug. 11, 1995; revised Jan. 16, 1996; accepted Jan. 25, 1996.

This work was supported by National Science Foundation Grants BNS8909620 and IBN9209490. We thank Melissa Bodner, Kelli Brice, and Lesley Turner for assistance in training the animals; David Grundy for computer programming; Judy Kodger and Elaine Brophy for animal training, video data analysis, and data collation; and Dr. Daniel Goldreich for comments on this manuscript.

Correspondence should be addressed to George E. Carvell, 6032 Forbes Tower, Department of Physical Therapy, School of Health and Rehabilitation Sciences, University of Pittsburgh, Pittsburgh, PA 15260.

Copyright $(\mathcal{C} 1996$ Society for Neuroscience $0270-6474 / 96 / 162750-08 \$ 05.00 / 0$
}

In previous studies, we have found that rats can use their vibrissae to distinguish subtle differences in surface texture and form (Carvell and Simons, 1990, 1995) (see also Guic-Robles et al., 1989). The mystacial vibrissae comprise an array of specialized hairs that is actively swept back and forth during tactile exploration and object palpation (Welker, 1964; Wineski, 1983; Carvell et al., 1991). Although whisker movements occur at a characteristic rhythm centered at $\sim 8 \mathrm{~Hz}$, whisking differs subtly but measurably in different animals. Recently, we found that movement patterns vary with the overall performance abilities of individual rats and with the nature of the surfaces they are palpating (Carvell and Simons, 1995).

The purpose of the present study is to investigate the behavioral consequences in adulthood of neonatal whisker trimming. Using a two-choice sensory discrimination paradigm and video-based biometric analyses, we examined the proficiency and movement strategies of infant-trimmed rats performing vibrissal discriminations using the regrown whiskers. Results were compared with previously studied normally reared animals and with animals whose whiskers were trimmed only during adulthood. Rats raised with shortened whisker hairs were profoundly impaired in their ability to perform some types of tactile discriminations even after months of training. Whisker movements were also abnormal. Abnormal tactile experience early in life thus appears to affect sensorimotor integration underlying active touch.

\section{MATERIALS AND METHODS}

Subjects. Three groups of Sprague-Dawley female rats were used in this study. Pregnant rats were procured during their last week of gestation from a local supplier (Zivic Miller, Allison Park, PA). At 12-24 hr after birth, infant-trimmed rats $(n=11)$ were gently restrained or briefly anesthetized with methoxyflurane vapors and all of their mystacial vibrissae on both sides of the face were clipped to within $2-3 \mathrm{~mm}$ of the skin surface; trimming continued daily to $45 \mathrm{~d}$ of age. All whiskers were subsequently allowed to regrow for $60-70 \mathrm{~d}$ before discrimination train- 
ing began. After this period of vibrissal regrowth, infant-trimmed animals that would be trained on the rough/smooth (RS) task ( $n=6$, see below) had all whiskers except those in the middle "C row" trimmed weekly to a length too short to contact the discriminanda $(\sim 1.0-1.5 \mathrm{~cm}$ in length) but of sufficient length to engage objects close to the face during natural exploratory behavior. The shortening was done to facilitate the video analyses and was not expected to affect the behavior because normal animals easily perform the RS task with only a single vibrissa (Carvell and Simons, 1995). This expectation was borne out by the infant-trimmed animals' behavior during training on the RS task. Animals trained on the rough/rough (RR) task $(n=5)$ had all of their vibrissae at full length during training. Adult-trimmed animals $(n=10)$ were obtained from the same supplier at $45 \mathrm{~d}$ of age. Under brief anesthesia, these animals had their whiskers clipped daily to within $2-3 \mathrm{~mm}$ of the skin surface for $45 \mathrm{~d}$; all vibrissae were allowed to regrow for $60-70 \mathrm{~d}$ before initiation of discrimination training. Normally reared animals $(n=18)$ were trained with no previous whisker clipping.

Bchavioral training. For a food reward, blindfolded animals were trained to perform texture discriminations using only their mystacial vibrissae. The use of removable blindfolds, the behavioral testing apparatus, and the training procedures have been described in detail in Carvell and Simons $(1990,1995)$. Each animal is trained to stand on an elevated "start" platform and stretch across a gap to palpate, with its outstretched vibrissae, discriminanda attached to the front of two "choice" platforms. Animals were trained to choose between a "reward" surface and a "nonreward" surface. The reward and nonreward discriminanda were randomly interchanged between the right and left choice platforms to discourage the formation of right/left biases. Animals designated their choice by jumping to one of the platforms. A correct choice was rewarded by the experimenter raising a gate to allow the animal access to a rat chow mash laced with peanut butter. An animal could sample each surface as many times as it chose but was not permitted to self-correct, i.e., jumping to the incorrect platform terminated the trial with no access to the food reward. Animals were trained $5 \mathrm{~d} /$ week for 10-14 trials/day. After the daily testing sessions, animals were fed rat chow pellets for $30 \mathrm{~min}$. All animals displayed weight gain during the study; animal weights ranged between 300 and $500 \mathrm{gm}$ during the course of training. Water was available ad libitum. Care and housing of the rats were in accordance with National Institutes of Health guidelines.

Discriminanda were constructed from high-density plastic cylinders (Delrin, DuPont NEN, Boston, MA) that were rounded and smoothed on a precision lathe. The smooth discriminandum had no further machining of its surface, whereas all rough discriminanda were subsequently turncd to produce the desired groove pattern. Animals were trained on one of two vibrissal discrimination tasks. In an RS paradigm, rats distinguished the smooth surface from one having finely cut grooves. A repeated pattern of grooves having equal widths, depths, and spacings at 500,250 , $200,150,100$, or $50 \mu \mathrm{m}$ was used to form the battery of rough discriminanda (Fig. $1 A$, see legend). The $\mathrm{RR}$ paradigm required animals to distinguish a $1 \mathrm{~mm}$ grooved standard (groove width, spacing, and depth = $1 \mathrm{~mm}$ ) from a discriminandum having wider grooves of equal widths and spacing $(2.00,1.75,1.50,1.25,1.125,1.06 \mathrm{~mm})$, all of which were $1 \mathrm{~mm}$ deep (Fig. $1 C$, see legend).

The initial stage of training was designed to teach the animals the basic task of palpating the surfaces and hopping onto the reward platform. Discriminanda consisted of surfaces having markedly different surface features. For the RS paradigm, this initial training paired the smooth discriminandum with one having wide and deep spiral grooves cut at 8 turns/inch. For the RR task, the $1 \mathrm{~mm}$ standard was paired with a surface having 3-mm-wide grooves spaced $3 \mathrm{~mm}$ apart. Once an animal showed evidence of understanding the nature of the task, the test discriminanda were introduced on half of the trials. For the RS task, this was the $500 \mu \mathrm{m}$ surface; for the RR task, this was the $2.0 \mathrm{~mm}$ surface. As reported below, all but one of the infant-trimmed RR animals were unable to learn even the initial task. They were removed from testing after $100 \mathrm{~d}$, by which time they had developed intractable left/right biases or no longer attended to the task.

Once discrimination testing commenced animals progressed to successively narrower gratings, e.g., from 500 to $250 \mu \mathrm{m}$ versus smooth (RS) or from 2.0 to $1.75 \mathrm{~mm}$ versus $1 \mathrm{~mm}$ (RR). Half of the trials contained the most recently mastered surface, the other half used the newly introduced one. Based on our previous experience with control animals, mastery of each surface pairing was defined as $>85 \%$ correct choices for three consecutive days with one day at $100 \%$ for the RS task or $>75 \%$ correct choices for three consecutive days for the RR task. Animals were re- moved from testing if they were unable to master a pairing within $25 \mathrm{~d}$, a period well above the average value observed previously in normally reared animals.

Analysis of whisking biometrics. Whisking behavior was analyzed using high-resolution videography and computer-assisted motion analysis (Peak Performance Technologies, Englewood, CO). Methods are described in detail in Carvell et al. (1991) and Carvcll and Simons (1995). Each trial was subdivided into an approach phase, as the animal whisked the air while stretching forward to palpate the discriminandum, and a contact phase, which commenced with the first observable whisker/surface contact. The contact phase ended when the animal moved forward to jump to the platform or retreated from the discriminandum. Only those trials in which the animal eventually made a correct choice were chosen for detailed biometric measurements; this could include palpation of the reward or nonreward surface. The positions of four large whiskers ( 2 on the right side and 2 on the left side of the face) were measured in each trial. Typically, these were chosen from whiskers 1 through 4 in rows B-D. Great care was taken to ensure that the same whiskers were measured for all consecutive video fields, and all trials were double-checked for accuracy. Whisker position was measured along the hair shaft at 5 consecutive $0.5 \mathrm{~cm}$ intervals beginning at the skin surface for each consecutive video field $(60 / \mathrm{sec})$. The location of each point was manually marked using an adjustable cursor.

The change in angular position of each point was calculated using Peak Performance softwarc. Further biometric measurements were made with our custom software and with Lotus. Data were subsequently analyzed with SPSS-PC+. Discrete Fourier power spectra $(2-30 \mathrm{~Hz})$ were computed for each whisker on a trial-by-trial basis, and average Fourier transforms were calculated across all whiskers and all trials for each animal. Sweep amplitudes and velocities were calculated for each whisker at the $1.0 \mathrm{~cm}$ marker in each trial. A whisking sweep was defined as movement of a whisker in the same direction for two or more consecutive fields. The amplitude for forward (protraction) and backward (retraction) sweeps was computed in degrees, and sweep vclocitics werc calculated in $\mathrm{dcg} / \mathrm{sec}$ using the following formula: angular motion $(\mathrm{deg}) /($ number of fields $\times 16.67 \mathrm{msec})$. For each vibrissa, the degree of whisker deformation (bending) was calculated as the difference in angular position of the whisker at 1.0 versus $2.5 \mathrm{~cm}$ from the base of the hair. A greater degree of bending is associated with a larger angular difference. Bending data were calculated only for those video fields in which the whisker contacted the discriminandum. Data were obtained from 13,226 whisking sweeps; approximately the same amount of data was collected from each animal.

\section{RESULTS}

\section{Discrimination performance}

Every animal learned the basic task of stretching across the gap, palpating the discriminanda with its vibrissae and jumping to one of the two choice platforms during discrimination testing. Infanttrimmed animals performed well on the RS task (Fig. 1B). The performance of these animals on the series of RS pairings was indistinguishable from that of the normal adults (Fig. $1 A$ ) and of adult-trimmed animals $(p>0.05$, repeated-measures ANOVA; adult-trimmed data not shown). Pooling across groups revcalcd that, on average, rats required $4.55 \mathrm{~d}$ to attain criterion performance on each of the pairings. All rats successfully completed the entire battery, including that of a smooth surface paired with one having grooves at $50 \mu \mathrm{m}$ intervals.

In the case of the RR task, normal animals needed an average of $10.71 \mathrm{~d}$ to progress from one pairing to the next (Fig. 1C). Two normal animals failed to master the 1.125 versus 1 mm pairing in the series during $25 \mathrm{~d}$ of training and were removed from further testing. One adult-trimmed animal failed to master the $1.25 \mathrm{~mm}$ discrimination and was not tested on the 1.125 or $1.06 \mathrm{~mm}$ surfaces. For surfaces eventually mastered, normal adult and adult-trimmed animals did not differ from each other on days to criterion (repeated-measures ANOVA, $p>0.05$ ).

Infant-trimmed animals trained on the RR task were severely impaired compared with normal and adult-trimmed rats in their 

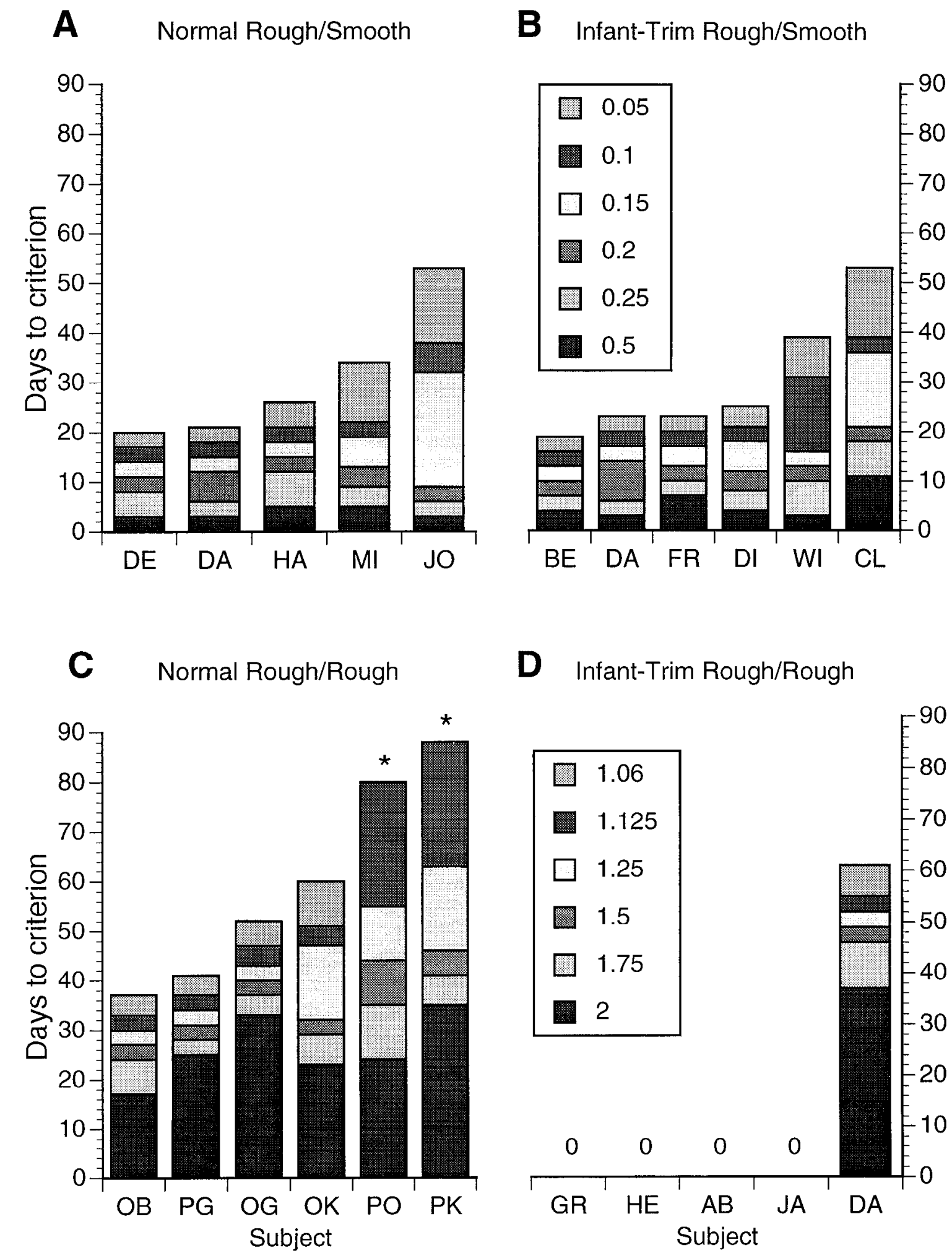

D Infant-Trim Rough/Rough

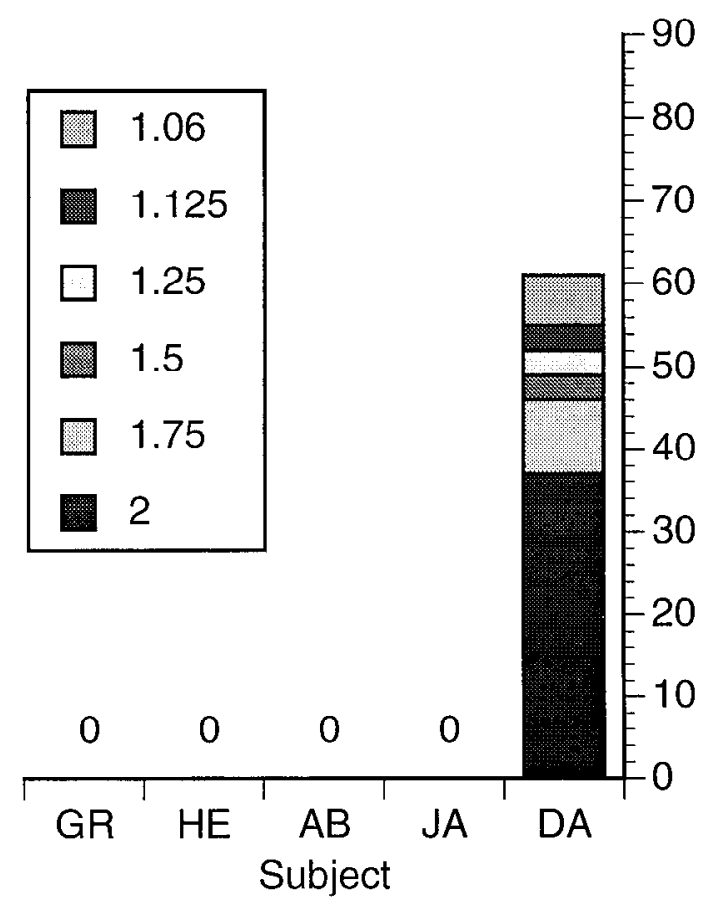

Figure 1. Performance of normally reared and infant-trimmed animals on the RS or RR task. The performance of each subject (denoted by its initials along the abscissa in each panel) is plotted as the number of days required to reach criterion for each $\mathrm{RS}(A, B)$ or $\mathrm{RK}(C, D)$ discrimination pairing. Stacked bars show performance for the sequential RS or RR pairings. Animals are ordered in each group according to their overall performance. Legends show the groove spacing (in $\mathrm{mm}$ ) for each discriminandum paired with either the smooth (RS task, $A, B$ ) or the $1.0 \mathrm{~mm}$ standard (RR task, $C, D$ ). Asterisks for $P O$ and $P K$ in $C$ indicate that the animal failed to attain criterion performance 1.125 versus $1.0 \mathrm{~mm}$ pairing. In $D, 0$ for $G R, H E, A B$, and $J A$ denotes the failure of these animals to learn the initial task. 
A

\section{APPROACH CONTACT}
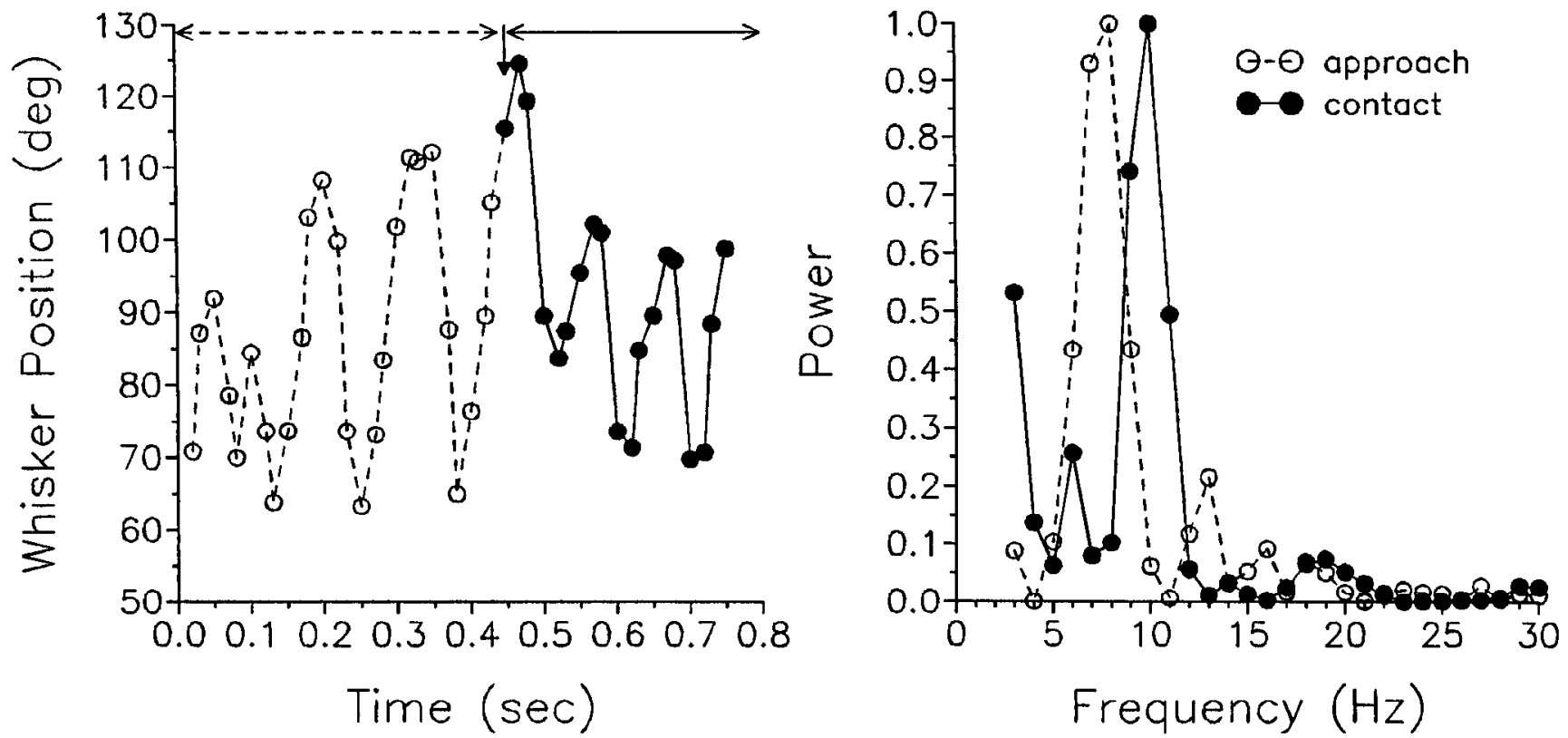

Figure 2. Whisking pattern changes on surface contact. $A$ shows the motion of a single whisker from a single trial of a normally reared RR animal as the animal approaches (open circles) and then contacts (filled circles) a discriminandum. Data points indicate successive angular position (in degrees) of the whisker on a field-by-field basis $(60$ fields/sec). Larger angles denote a more protracted position. $B$ plots the Fourier power spectrum for this whisker during the approach phase (open circles) and contact phase (filled circles).

ability to perform the RR task (Fig. $1 D$ ). Only one subject (DA) was able to complete the series. Despite $100 \mathrm{~d}$ of training, the remaining four infant-trimmed subjects were unable to master even the initial pairing ( $3.0 \mathrm{vs} 1.0 \mathrm{~mm}$ ) and, thus, failed to progress to other problems in the series. Thus, all normally reared $(n=6)$ and all adult-trimmed animals learned at least the first surface pairing in the series, whereas only one of five infant-trimmed animals did. Fisher's exact tests revealed significant differences between the infant-trimmed group and each of the others $(p<$ $0.05)$.

\section{Whisking biometrics}

We examined videotaped sequences of the animals' whisking behavior to determine whether whisker deprivation affects how animals use their vibrissae. Figure 2 illustrates a representative sequence from a normal adult animal performing the RR task. Figure $1 A$ is a reconstruction of the forward (protraction) and backward (retraction) movements of one whisker during a single trial. During "approach," the animal is moving its head forward to contact the discriminandum with its vibrissae; the whiskers are simply moving through air without any object contact as the animal stretches across the gap between the start and choice platforms. Once contact occurs, the whisking becomes somewhat more rapid and of smaller amplitude. Active whisker protractions (movements from smaller to larger angular values in Fig. $1 A$ ) are slower than retractions. A previous study has demonstrated that protractions are actively generated by a burst of activity in mystacial pad musculature, whereas retractions result largely from passive elastic recoil (Carvell et al., 1991). Fourier power spectra of these data are shown in Figure $1 B$. Whisking is strongly characterized by frequencies in the range of $6-12 \mathrm{~Hz}$, and the dominant frequency shifts from $8 \mathrm{~Hz}$ during approach to $10 \mathrm{~Hz}$ during actual palpation of the discriminandum (see also below).

The frequency characteristics of whisking during actual object contact are shown for normal and infant-trimmed animals in Figure 3. Figure $3, A, B, D$, and $E$, shows power spectrum data from individual animals performing the RS or RR tasks; averages are plotted in Figure 3, $C$ and $F$. Whisking movements of normal subjects were strongly dominated by frequencies in the $6-12 \mathrm{~Hz}$ range, regardless of task. Highly similar patterns were observed in the infant-trimmed animals that successfully mastered their respective task, that is, all RS- and one RR-trained animal. Whisker movements of the (4 RR-trained) infant-trimmed animals that failed their task, however, had substantial contributions of frequencies above and below the $6-12 \mathrm{~Hz}$ range, with a relative absence of power within it (Fig. $3 E, F$ ).

For infant-trimmed RS animals, whisking frequency characteristics during approach were also similar to those of normal subjects. Figure $4, A$ and $B$, illustrates that both groups of animals have power spectra that are clearly dominated by a single, narrow range of frequencies that shifts upward during object contact. All five infant-trimmed RR animals displayed abnormal whisking during approach. Their power spectra were bimodal, with dominant frequencies bracketing the normal $6-12 \mathrm{~Hz}$ range. Interestingly, DA, the one infant-trimmed RR animal that learned the task, altered its whisking pattern after surface contact such that it used frequency components in the normal range during object 


\section{Rough vs Smooth}
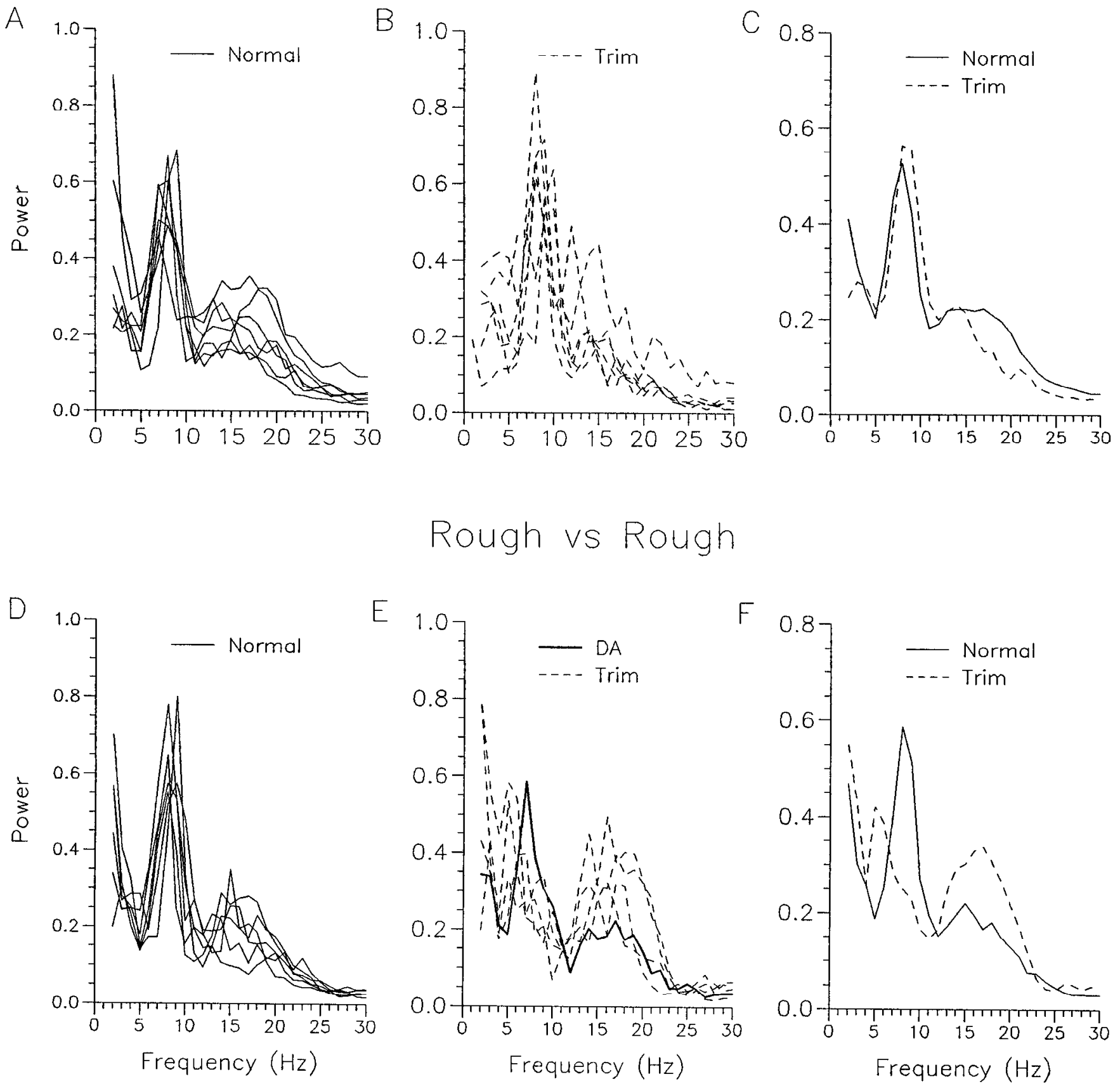

Figure 3. Whisking frequency of normally reared and infant-trimmed rats. A discrete Fourier transform (bandwidth 2-30 $\mathrm{Hz}$ ) was computed for each animal's whisking pattern during palpation of a discriminandum. $A, B, D$, and $E$ show averaged power spectra for each animal; the average is based on the frequency analyses of four whiskers across all trials. In $E$, data from the sole infant-trimmed animal that learned the RR task $(D A)$ is highlighted with a solid line. $C$ and $F$ show power spectra averaged across all animals for the normally reared and infant-trimmed groups; data from DA were excluded from the RR average. Fourier power spectra for the normally reared RS animals are based on data from a previously analyzed group of rats (see Carvell and Simons, 1995).

palpation. The power spectra of the other infant-trimmed RR animals remained abnormal during surface palpation.

Figure 5 shows three other measures of whisking motion. Values were obtained by first computing means for each animal and then averaging these to oblain a group score. For both tasks, movement velocities of infant-trimmed animals were comparable with those of normally reared animals (Mann-Whitney $U$ tests, $p>0.05$ ). Infant-trimmed RS, but not RR, subjects used abnormally large amplitude whisking sweeps $(p=0.01)$ and displayed correspondingly larger than normal whisker deformation compared with their normally reared counterparts $(p<0.05)$.

As noted above, the infant-trimmed animals performing the RS 
A

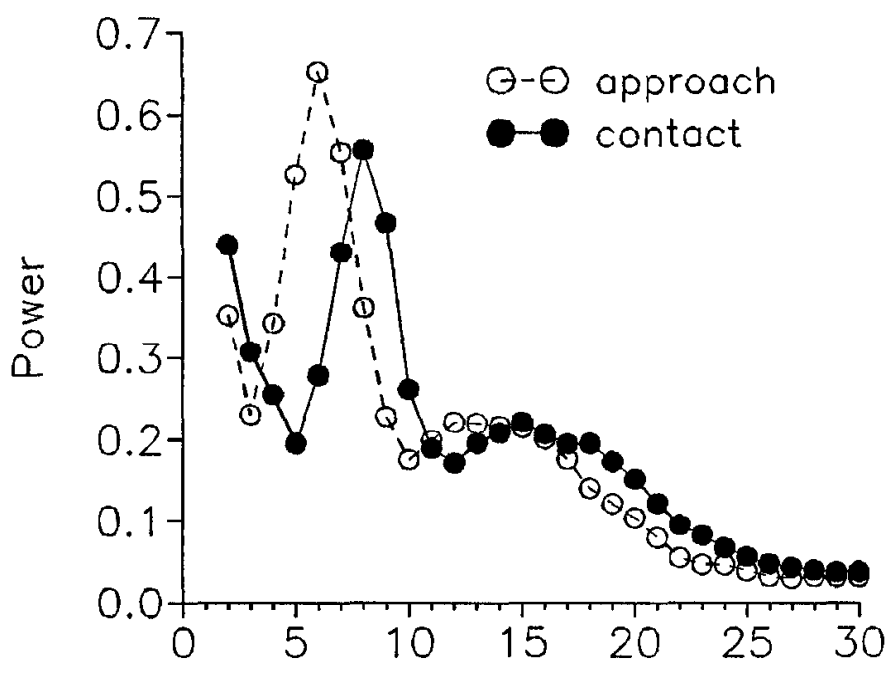

B Infant-Trim Rough/Smooth

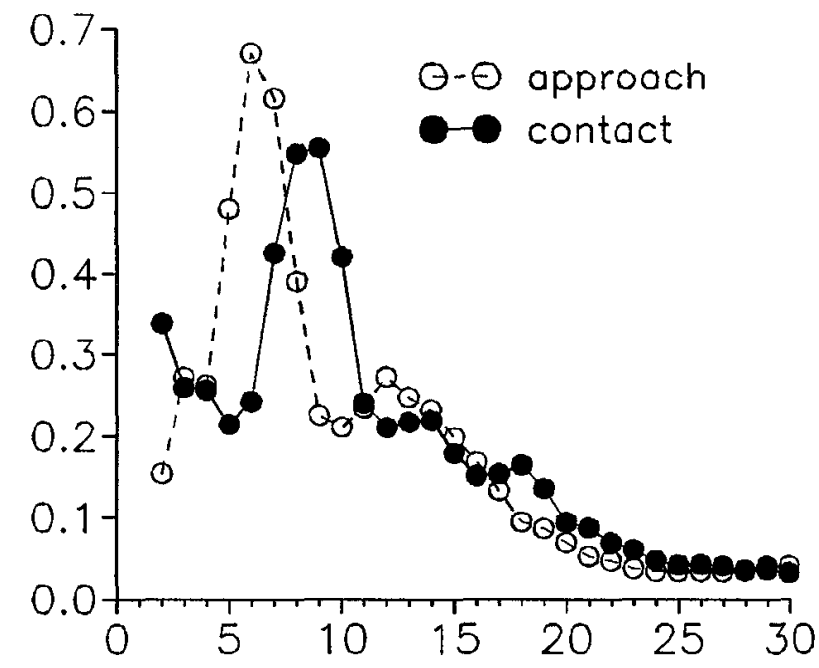

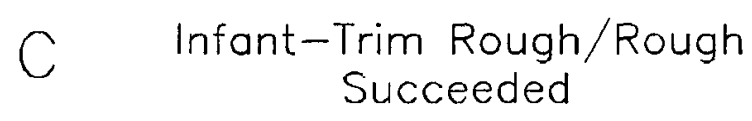

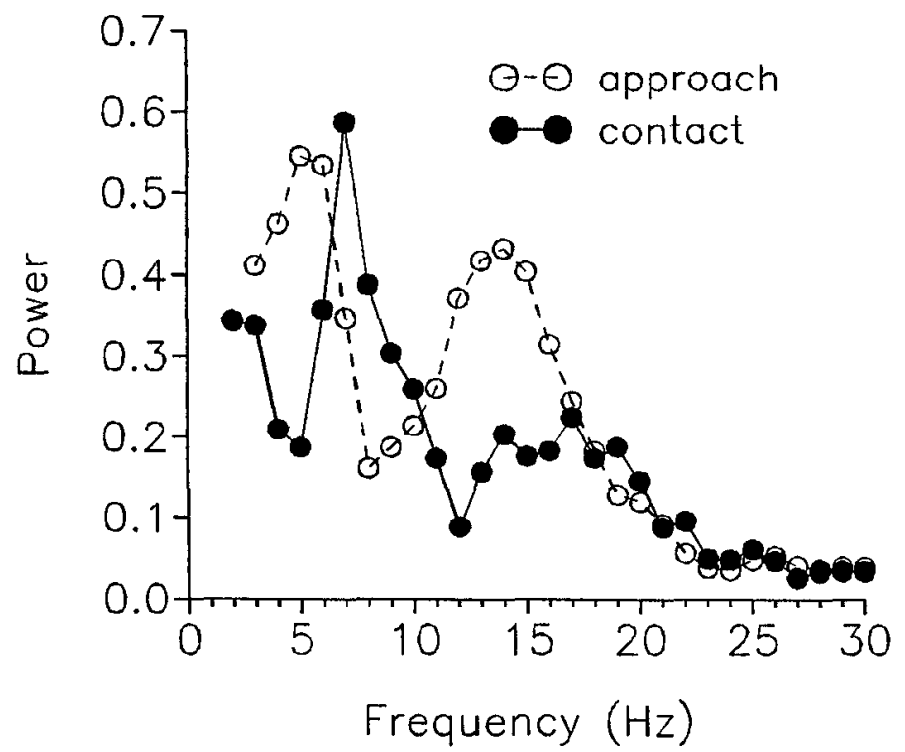

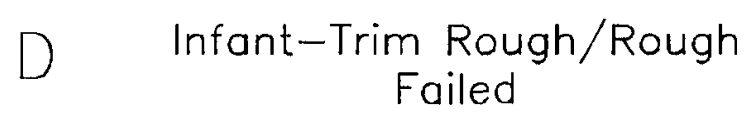

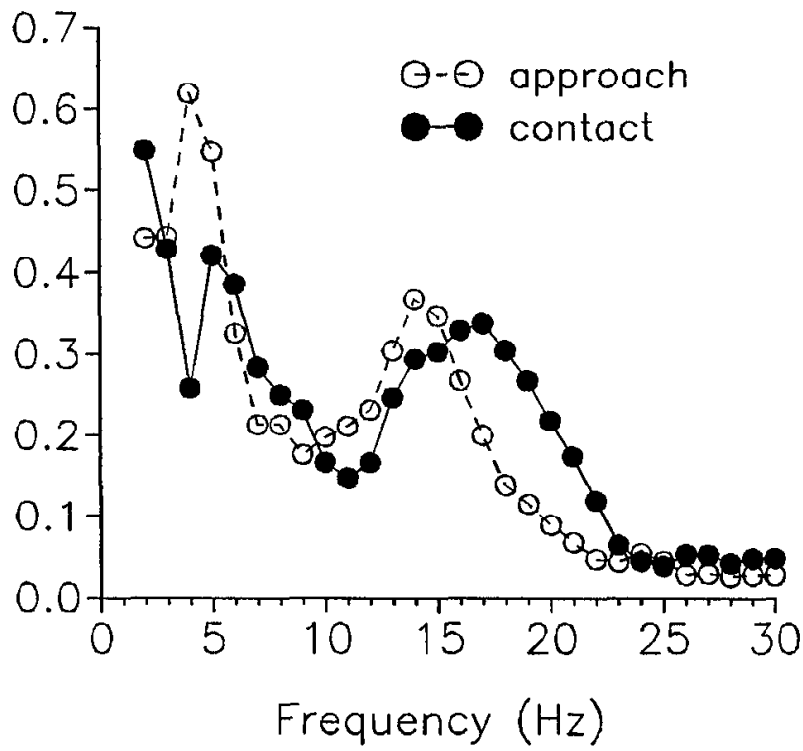

Figure 4. Approach to contact phase Fourier power spectra shifts for normal and infant-trimmed animals performing the RS or RR task. Fourier power spectra are plotted for the approach (open circles) and contact (filled circles) phases averaged across four whiskers ( 2 right, 2 left) for all trials. $A$ plots data combined for all normally reared animals ( $7 \mathrm{RS}$ and $6 \mathrm{RR}$ animals). $B$ plots data from six infant-trimmed RS animals, and data from the infant-trimmed RR animals are shown in $C$ and $D$. $C$ shows the power spectra for the one animal that learned the RR task; $D$ shows data from the remaining four animals that never learned the RR task.

task had thcir non-row $\mathrm{C}$ whiskers shortened to facilitate video analyses. Because our normal RS sweep data are based on discriminations performed by animals with all whiskers intact, we compared sweep data from two normal animals that were videotaped performing the RS task while using all vibrissae and when all but the $\mathrm{C}$ row of whiskers were shortened. For one animal, sweep amplitude and whisker deformations were the same in both conditions ( $t$ tests, $p>0.05$ ). The other animal used smaller amplitude sweeps with somewhat less whisker bending when only one row of whiskers was intact ( $t$ tests, $p<0.05$ ). This was opposite from what was observed in the infant-trimmed animals. Thus, the shortening of the hairs is unlikely to have contributed to the observed differences between the normally reared and infanttrimmed values. 
A

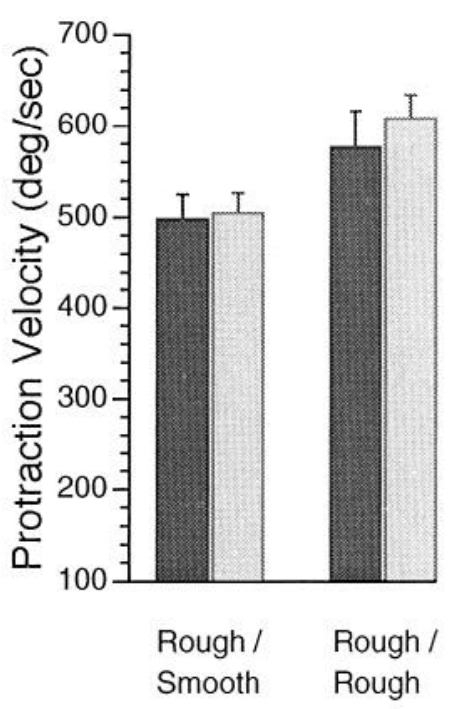

B

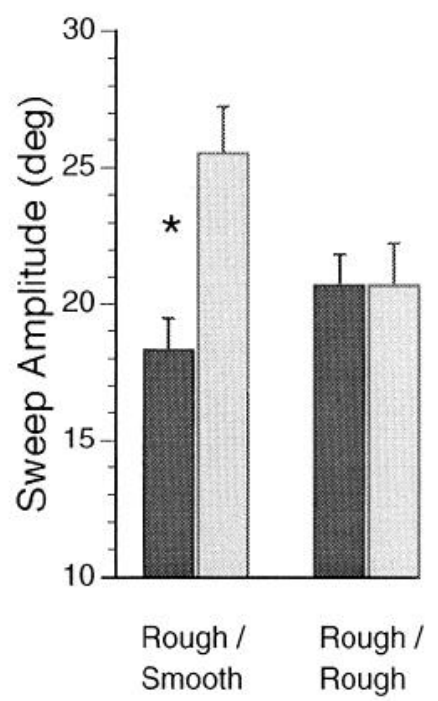

C

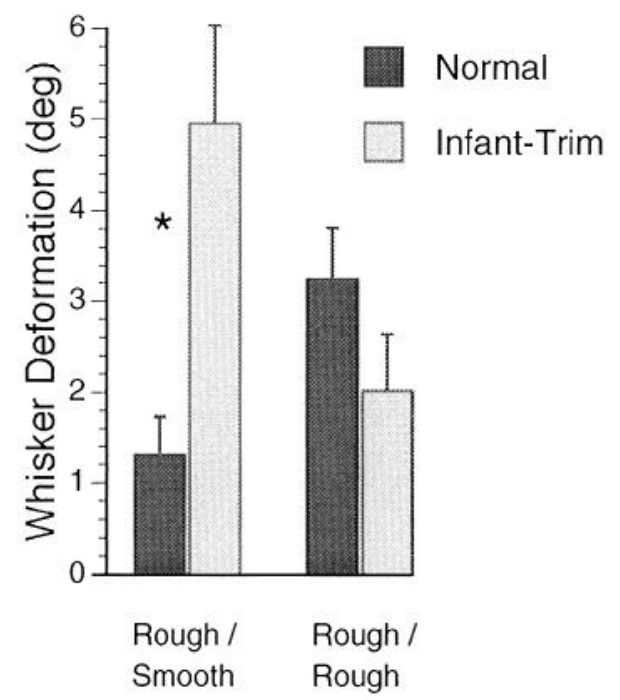

Figure 5. Biometrics of whisking sweeps in normally reared and infant-trimmed animals. The means and SEM for protraction velocities in deg/sec $(A)$, sweep amplitude in deg $(B)$ and whisker deformation (bending) in deg $(C)$ are shown for normally reared RS $(n=7)$ and RR $(n=6)$ animals (see Carvell and Simons, 1995) and for their infant-trimmed counterparts (RS, $n=6 ; \mathrm{RR}, n=5)$. An asterisk denotes a statistical difference between the normal and infant-trimmed groups for the designated biometric measure (see text).

\section{DISCUSSION}

The present findings demonstrate that whisker trimming in young, but not mature, rats leads to pronounced deficits in whisker-based sensory discrimination. Deficits are task-dependent. Infanttrimmed animals learned the RS discriminations as rapidly as normally reared rats and adult-trimmed subjects. Once learned, these same discriminations can be performed by normally reared animals using only a single whisker (Carvell and Simons, 1995). By contrast, four of five infant-trimmed animals were unable to master the RR discriminations. In normally reared rats, these tasks require the presence of at least two whiskers (on each side of the face). Thus, the two types of tasks appear to tap into different domains of information processing in the whisker system, one of which depends critically on early sensory experience. The precise nature of such experience remains to be determined, but one straightforward hypothesis, based on available data, is that more than one long whisker must be present postnatally if animals are to perform the RR task successfully as adults.

The aforementioned differences in the ability of normally reared animals to perform discriminations using single versus multiple whiskers suggest that the deficits displayed by infanttrimmed animals on the RR, but not RS, task reflect an inability to integrate in an appropriate manner information from neighboring whiskers. Neurons in layer IV of the adult somatosensory cortex of infant-trimmed animals are known to respond to deflections of regrown whiskers with abnormally vigorous and prolonged discharges, to include a larger number of whiskers within their excitatory receptive fields, and to display weaker inhibitory interactions among inputs from neighboring vibrissae (Simons and Land, 1987; Simons et al., 1993). Thus, the behavioral abnormalities displayed by infanttrimmed animals may reflect loss or diminution of spatial and/or temporal contrast within the receptive fields of cortical neurons. A pivotal role for processing at the cortical level is supported by the findings that response properties of thalamic neurons are largely normal in infant-trimmed animals (Simons and Land, 1994) and that an intact somatosensory cortex is required for the performance of vibrissal texture discriminations in otherwise normal adult rats (Guic-Robles et al., 1992).

Infant-trimmed RS animals performed their task well despite the use of abnormally large whisking sweeps that in turn produce abnormally large whisker deformations. These findings indicate that neonatal whisker trimming was unlikely to have produced atrophy of the whisker pad musculature. Four of the infant-trimmed RR animals failed to master their task even though their whisking amplitudes, velocities, and bending values were within the ranges displayed by normal subjects. On the other hand, all infant-trimmed animals that learned their task (all RS and $1 \mathrm{RR}$ ) had whisking power spectra that contained pronounced $6-12 \mathrm{~Hz}$ components during object contact, whereas none of the unsuccessful (RR) animals did. Our previous studies of normal rats have shown that better performers on either the RS or RR tasks use whisking patterns that are more strongly dominated by frequencies in the $6-12$ $\mathrm{Hz}$ range (Carvell and Simons, 1990, 1995). Because in infanttrimmed animals whisking frequency spectra are strongly correlated with the nature of the sensory discrimination task (RR vs RS), it appears that the abnormal frequencies displayed by $\mathrm{RR}$ animals reflect a deprivation-induced deficit in sensory processing. This could be attributable to the motor system's receipt of aberrant signals from sensory cortex (Sakamoto et al., 1989; Iriki et al., 1991) (see also Sharp and Evans, 1982). In this regard, neurons located superficial and deep to the barrels are known to project to vibrissa motor cortex (Donoghue and Parham, 1983; Porter and White, 1983; Miyashita et al., 1994). According to this hypothesis, an infant-trimmed rat trained on both the RS and the RR tasks would display normal and abnormal whisking patterns, depending on which task it is performing.

Simply clipping a rat's whiskers for several weeks during early 
postnatal life leads to profound and apparently permanent alterations in its somatosensory system. Electrophysiological examination of three of the infant-trimmed subjects in the present study (1 RS, 2 RR) demonstrated abnormalitics in the responsc propertics of their cortical neurons despite months of whisker regrowth and behavioral training (Simons et al., 1993). These findings underscore the importance of experience in brain development and the age dependence of the response of the nervous system to it.

\section{REFERENCES}

Akhtar ND, Land PW (1991) Activity-dependent regulation of glutamic acid decarboxylase in the rat barrel cortex: effects of neonatal versus adult sensory deprivation. J Comp Neurol 307:200-213.

Carvell GE, Simons DJ (1988) Membrane potential changes in rat SmI cortical neurons evoked by controlled stimulation of mystacial vibrissae. Brain Res 448:186-191.

Carvell GE, Simons DJ (1990) Biometric analyses of vibrissal tactile discrimination in the rat. J Neurosci 10:2638-2648.

Carvell GE, Simons DJ (1995) Task- and subject-related differences in sensorimotor behavior during active touch. Somatosens Mot Res 12:1-9.

Carvell GE, Simons DJ, Lichtenstein SH, Bryant P (1991) Electromyographic activity of mystacial pad musculature during whisking behavior in the rat. Somatosens Mot Res 8:159-164.

Donoghue JP, Parham C (1983) Afferent connections of the lateral agranular field of the rat motor cortex. J Comp Neurol 217:390-404.

Fox K (1992) A critical period for experience-dependent synaptic plasticity in rat barrel cortex. J Neurosci 12:1826-1838.

Guic-Robles E, Valdivieso C, Guajardo G (1989) Rats can learn a roughness discrimination using only their vibrissal system. Behav Brain Res 31:285-289.

Guic-Kobles E, Jenkins WM, Bravo H (1992) Vibrissal roughness dis crimination is barrelcortex dependent. Behav Brain Res 48:145-152.

Hubel DH, Wiesel TN (1970) The period of susceptibility to the physiological effects of unilateral eye closure in kittens. J Physiol (Lond) 206:419-436.
Hubel DH, Wiesel TN, LeVay S (1977) Plasticity of ocular dominance columns in monkey striate cortex. Phil Trans R Soc Lond [Biol] 278:377-409.

Iriki A, Pavlides C, Keller A, Asanuma H (1991) Long-term potentiation of thalamic input to the motor cortex induced by coactivation of thalamocortical and corticocortical afferents. J Neurophysiol 65:1435-1441.

Mitchell DE, Timney B (1984) Postnatal development of function in the mammalian visual system. In: Handbook of physiology-the nervous system III (Darian-Smith I, ed), pp 507-5.55. Bethesda: American Physiological Society.

Miyashita E, Keller A, Asanuma H (1994) Input-output organization of the rat vibrissal motor cortex. Exp Brain Res 99:223-232.

Porter LL, White EL (1983) Afferent and efferent pathways of the vibrissal region of primary motor cortex in the mouse. $J$ Comp Neurol 214:279-289.

Sakamoto T, Arissian K, Asanuma H (1989) Functional role of the sensory cortex in learning motor skills in cats. Brain Res 503:258-264.

Sharp HR, Evans K (1982) Regional $\left({ }^{14} \mathrm{C}\right)$ 2-deoxyglucose uptake during vibrissae movements evoked by rat motor cortex stimulation. J Comp Neurol 208:255-287.

Simons DJ (1985) Temporal and spatial integration in the rat SI vibrissa cortex. J Neurophysiol 54:615-635.

Simons DJ, Carvell GE (1989) Thalamocortical response transformation in the rat vibrissa/barrel system. J Neurophysiol 61:311-330.

Simons DJ, Land PW (1987) Early experience of tactile stimulation influences organization of somatic sensory cortex. Nature 326:694-697.

Simons DJ, Land PW (1994) Neonatal whisker trimming produces greater effects in non-deprived than deprived thalamic barreloids. J Neurophysiol 72:1434-1437.

Simons DJ, Land PW, Suter K (1993) Neonatal whisker trimming reduces surround inhibition in adult barrel cortex. Soc Neurosci Abstr 19:46.

Welker WI (1964) Analysis of sniffing of the albino rat. Behaviour 22:223-244.

Wineski LE (1983) Movements of the cranial vibrissae in the golden hamster (Mesocricetus auratus). J Zool 200:261-280. 